\title{
Comparison of admission random glucose, fasting glucose, and glycated hemoglobin in predicting the neurological outcome of acute ischemic stroke: A retrospective study
}

\author{
Jia-Ying Sung ${ }^{1,2}$, Chin-I Chen ${ }^{1,2}$, Yi-Chen Hsieh ${ }^{3}$, Yih-Ru Chen ${ }^{4}$, Hsin-Chiao Wu ${ }^{4}$, Lung Chan ${ }^{2,5}$, Chaur- \\ Jong Hu ${ }^{2,5}$, Han-Hwa Hu ${ }^{2,5}$, Hung-Yi Chiou ${ }^{4}$, Nai-Fang Chi ${ }^{\text {Corresp. } 2,5}$ \\ 1 Department of Neurology, Wang Fang Hospital, Taipei, Taiwan \\ Department of Neurology, School of Medicine, College of Medicine, Taipei Medical University, Taipei, Taiwan \\ 3 The PhD Program of Neural Regenerative Medicine, College of Medical Science and Technology, Taipei Medical University, Taipei, Taiwan \\ 4 School of Public Health, College of Public Health, Taipei Medical University, Taipei, Taiwan \\ 5 Department of Neurology, Shuang Ho Hospital, New Taipei City, Taiwan \\ Corresponding Author: Nai-Fang Chi \\ Email address: naifangchi@tmu.edu.tw \\ Background. Hyperglycemia is a known predictor of negative outcomes in stroke. Several glycemic \\ measures, including admission random glucose, fasting glucose, and glycated hemoglobin (HbAlc), have \\ been associated with bad neurological outcomes in acute ischemic stroke, particularly in nondiabetic \\ patients. However, the predictive power of these glycemic measures is yet to be investigated.
}

Methods. This retrospective study enrolled 484 patients with acute ischemic stroke from January 2009 to March 2013, and complete records of initial stroke severity, neurological outcomes at 3 months, and glycemic measures were evaluated. We examined the predictive power of admission random glucose, fasting glucose, and $\mathrm{HbAlc}$ for neurological outcomes in acute ischemic stroke. Furthermore, subgroup analyses of nondiabetic patients and patients with diabetes were performed separately.

Results. Receiver operating characteristic (ROC) analysis revealed that admission random glucose and fasting glucose were significant predictors of poor neurological outcomes, whereas HbAlc was not [areas under the ROC curve (AUCs): admission random glucose $=0.564, p=0.026$; fasting glucose $=0.598, p$ $=0.001 ; \mathrm{HbAlc}=0.510, p=0.742 \mathrm{]}$. Subgroup analyses of nondiabetic patients and those with diabetes revealed that only fasting glucose predicts neurological outcomes in patients with diabetes, and the AUCS of these three glycemic measures did not differ between the two groups. A multivariate logistic regression analysis of the study patients indicated that only age, initial stroke severity, and fasting glucose were independent predictors of poor neurological outcomes, whereas admission random glucose and $\mathrm{HbAlc}$ were not (adjusted odds ratio: admission random glucose $=1.002, p=0.228$; fasting glucose $=1.005, p=0.039 ; \mathrm{HbAlc}=1.160, p=0.076)$. Furthermore, subgroup multivariate logistic regression analyses of nondiabetic patients and those with diabetes indicated that none of the three glycemic measures were associated with poor neurological outcomes.

Discussion. Fasting glucose is an independent predictor of poor neurological outcomes in patients with acute ischemic stroke and had greater predictive power than that of admission random glucose and $\mathrm{HbAlc}$. The predictive power of glycemic measures for poor neurological outcomes did not differ significantly between the nondiabetic patients and those with diabetes. 


\section{Title}

2 Comparison of admission random glucose, fasting glucose, and glycated hemoglobin in

3 predicting the neurological outcome of acute ischemic stroke- A retrospective study

4

\section{Short Title}

6 Hyperglycemia in acute ischemic stroke

\section{Authors}

9 Jia-Ying Sung ${ }^{1,2}$, Chin-I Chen ${ }^{1,2}$, Yi-Chen Hsieh ${ }^{3}$, Yih-Ru Chen ${ }^{4}$, Hsin-Chiao Wu ${ }^{4}$, Lung Chan ${ }^{2,5}$, Chaur-Jong $\mathrm{Hu}^{2,5}$, Han-Hwa $\mathrm{Hu}^{2,5}$, Hung-Yi Chiou${ }^{4}$, and Nai-Fang $\mathrm{Chi}^{2,5}$

11

12

13

14

15 16

21

22 Correspondence to:

23 Nai-Fang Chi

24 Department of Neurology, Taipei Medical University Shuang Ho Hospital, 25 291, Zhongzheng Road, Zhonghe District, New Taipei City, Taiwan.

26 E-mail address: naifangchi@tmu.edu.tw

${ }^{1}$ Department of Neurology, Wang Fang Hospital, Taipei Medical University, Taipei, Taiwan

${ }^{2}$ Department of Neurology, School of Medicine, College of Medicine, Taipei Medical University, Taipei, Taiwan

${ }^{3}$ The PhD Program of Neural Regenerative Medicine, College of Medical Science and Technology, Taipei Medical University, Taipei, Taiwan Taiwan ${ }^{4}$ School of Public Health, College of Public Health, Taipei Medical University, Taipei, Taiwan ${ }^{5}$ Department of Neurology, Shuang Ho Hospital, Taipei Medical University, New Taipei City, 
29

30

31

32

33

34

35

36

37

38

\section{ABSTRACT}

Background. Hyperglycemia is a known predictor of negative outcomes in stroke. Several glycemic measures, including admission random glucose, fasting glucose, and glycated hemoglobin (HbA1c), have been associated with bad neurological outcomes in acute ischemic stroke, particularly in nondiabetic patients. However, the predictive power of these glycemic measures is yet to be investigated.

Methods. This retrospective study enrolled 484 patients with acute ischemic stroke from January 2009 to March 2013, and complete records of initial stroke severity, neurological outcomes at 3 months, and glycemic measures were evaluated. We examined the predictive power of admission random glucose, fasting glucose, and $\mathrm{HbA1c}$ for neurological outcomes in acute ischemic stroke. Furthermore, subgroup analyses of nondiabetic patients and patients with diabetes were performed separately.

Results. Receiver operating characteristic (ROC) analysis revealed that admission random glucose and fasting glucose were significant predictors of poor neurological outcomes, whereas HbA1c was not [areas under the ROC curve (AUCs): admission random glucose $=0.564, p=0.026$; fasting glucose $=0.598, p=0.001 ; \mathrm{HbA1} c=0.510, p=0.742]$. Subgroup analyses of nondiabetic patients and those with diabetes revealed that only fasting glucose predicts neurological outcomes in patients with diabetes, and the AUCs of these three glycemic measures did not differ between the two groups. A multivariate logistic regression analysis of the study patients indicated that only age, initial stroke severity, and fasting glucose were independent predictors of poor neurological outcomes, whereas admission random glucose and HbAlc were not (adjusted odds ratio: admission random glucose $=1.002, p=0.228$; fasting glucose $=1.005, p=0.039 ; \mathrm{HbA} 1 \mathrm{c}=1.160$, $p=0.076$ ). Furthermore, subgroup multivariate logistic regression analyses of nondiabetic patients and those with diabetes indicated that none of the three glycemic measures were associated with poor neurological outcomes.

Discussion. Fasting glucose is an independent predictor of poor neurological outcomes in patients with acute ischemic stroke and had greater predictive power than that of admission random glucose and HbA1c. The predictive power of glycemic measures for poor neurological outcomes did not differ significantly between the nondiabetic patients and those with diabetes. 


\section{Introduction}

64 Hyperglycemia may be present in more than half of patients with acute ischemic stroke (Scott et

65 al., 1999). It is associated with an increased risk of infarction volume growth and hemorrhagic

66 transformation (Paciaroni et al., 2009; Parsons et al., 2002), a decreased recanalization rate of

67 thrombolytic therapy (Ribo et al., 2005), and an increased risk of poor neurological outcomes and

68 stroke mortality (Capes et al., 2001). Several indices of hyperglycemia have been reported in

69 stroke: elevated fasting glucose (Yao et al., 2016), elevated admission random glucose (Ahmed et

70 al., 2010; Ribo et al., 2005), maximum glucose during the acute disease stage (Fuentes et al., 2009),

71 and elevated glycated hemoglobin (HbA1c) (Kamouchi et al., 2011; Lattanzi et al., 2016). The

72 levels of fasting glucose, admission random glucose, and maximum glucose have been associated

73 with the stress response of stroke, particularly in nondiabetic patients (Capes et al., 2001; Stead et

74 al., 2009), and HbA1c was considered as a marker for prestroke glycemic control (Kamouchi et

75 al., 2011). Moreover, some studies have reported that in acute ischemic stroke, hyperglycemia is

76 more detrimental in nondiabetic patients than in those with diabetes (Capes et al., 2001; Stead et

77 al., 2009; Yao et al., 2016). Although these glycemic measures have been associated with

78 neurological outcomes in acute ischemic stroke, the most predictive factor among these is yet to

79 be reported. The present study compared the predictive performance of admission random glucose,

80 fasting glucose, and $\mathrm{HbA} 1 \mathrm{c}$ for poor neurological outcomes in acute ischemic stroke. Furthermore, 
81 the predictive performance of these glycemic measures was evaluated through subgroup analysis

82 of patients with diabetes and nondiabetic patients.

83

\section{Materials \& Methods}

\section{Study patients}

86 The present study was approved by the Joint Institutional Review Board of Taipei Medical

87 University (No. 201304008). Data were obtained by reviewing medical records of the patients with

88 acute ischemic stroke admitted to the hospitals affiliated with Taipei Medical University, Wan

89 Fang Hospital and Shuang Ho Hospital, between January 2009 and March 2013. Data were

90 included for analysis if the patients were aged $\geq 20$ years, presented to the emergency department

91 with acute ischemic stroke and were subsequently admitted to the neurology department, and

92 complete records of admission random glucose, fasting glucose, HbA1c, National Institute of

93 Health Stroke Scale (NIHSS) scores at admission, and modified Rankin Scale (mRS) scores at 3

94 months after stroke were obtained. After reviewing the enrolled medical records, patients with a

95 history of stroke or who died or were lost to follow-up within 3 months after stroke were excluded.

\section{Definitions of clinical characteristics and neurological outcomes}

98 In the present study, acute ischemic stroke was confirmed by clinical symptoms and neuroimages. 
99 The etiologic subtype of acute ischemic stroke was classified according to the Trial of Org 10172

100 in Acute Stroke Treatment (TOAST) study (Adams et al., 1993). Admission random glucose was

101 the first nonfasting plasma glucose measured at the emergency department. Fasting plasma glucose

102 and HbA1c were measured on the first morning of admission after at least 8 hours of fasting.

103 Hypertension was defined as a systolic blood pressure $>140 \mathrm{mmHg}$, a diastolic blood pressure $>$

$10490 \mathrm{mmHg}$, or current use of antihypertensive medicines. Diabetes was defined as HbA1c $\geqq 6.5 \%$

105 or current use of antidiabetic medicines. Hyperlipidemia was defined as total cholesterol $>200$

$106 \mathrm{mg} / \mathrm{dL}$ or current use of cholesterol-lowering medicines. Atrial fibrillation was diagnosed

107 according to either a documented history or an electrocardiogram on admission. Initial stroke

108 severity was evaluated on the first day of admission on the basis of the NIHSS at admission, and

109 neurological outcomes were evaluated on the basis of the mRS at 3 months after stroke. Poor

110 neurological outcomes were defined as an $\mathrm{mRS}$ score $=3$, 4, or 5 at 3 months after stroke.

\section{Statistical analyses}

113 The continuous variables, rank variables, and proportions of categorical variables were compared

114 between the nondiabetic patients and those with diabetes by using the Student t, Mann-Whitney

$115 \mathrm{U}$, and chi-squared tests, respectively. Receiver operating characteristic (ROC) analysis was

116 conducted for evaluating the predictive performance of the glycemic measures, namely admission 
117 random glucose, fasting glucose, and $\mathrm{HbA1c}$, in distinguishing between the favorable and poor

118 neurological outcomes. Specifically, the predictive performance of these glycemic measures was

119 evaluated by comparing their areas under the ROC curve (AUCs) (DeLong et al., 1988). A

120 univariate logistic regression analysis was conducted to estimate the odds ratio (OR) of poor

121 neurological outcomes for each variable. Age, sex, and variables with $p \leqq 0.2$ in the univariate

122 logistic regression analysis were included in a multivariate logistic regression model to adjust the

123 OR of poor neurological outcomes for the glycemic measures. Parametric and nonparametric data

124 were expressed as the mean \pm standard deviation (SD) and as the median with the interquartile

125 range (IQR), respectively, and $p<0.05$ was considered statistically significant. Statistical data

126 were analyzed using PASW Statistics for Windows version 18.0 (SPSS Inc, Chicago, IL, USA)

127 and MedCalc Statistical Software version 16.8 (MedCalc Software bvba, Ostend, Belgium,

128 https://www.medcalc.org, 2016).

129

130 Results

131 Table 1 summarizes the clinical characteristics of the study patients. Of the 484 patients enrolled

132 in the present study, 212 were nondiabetic and 272 had diabetes. Age, the proportion of

133 hypertension, admission random glucose, and fasting glucose in the patients with diabetes were

134 significantly higher than those in the nondiabetic patients, and the NIHSS score at admission, mRS 
135 score at 3 months after stroke, and proportions of poor neurological outcomes and the stroke

136 etiology subtype did not differ between the two groups.

137 The AUCs of admission random glucose, fasting glucose, and HbAlc in all patients are

138 presented in Figure 1A. In all patients, admission random glucose and fasting glucose were

139 significant predictors of poor neurological outcomes [AUCs: admission random glucose $=0.564$,

$14095 \%$ confidence interval $(\mathrm{CI})=0.519-0.609, p=0.026$; fasting glucose $=0.598,95 \% \mathrm{CI}=0.553-$

$1410.642, p=0.001]$; however, HbA1c was not a significant predictor $(\mathrm{AUC}=0.510,95 \% \mathrm{CI}=0.465-$

$1420.556, p=0.742$ ). The cutoff values with the highest sensitivity and specificity were $127 \mathrm{mg} / \mathrm{dL}$

143 for admission random glucose (sensitivity $=74.6 \%$, specificity $=39.3 \%$ ) and $109 \mathrm{mg} / \mathrm{dL}$ for

144 fasting glucose (sensitivity $=67.8 \%$, specificity $=51.6 \%$ ). The AUCs of admission random

145 glucose and fasting glucose did not differ significantly (0.564 vs. $0.598, p=0.194)$, and the AUCs

146 of admission random glucose and fasting glucose were significantly higher than that of HbA1c

147 (0.564 vs. $0.510, p=0.038$ and 0.598 vs. $0.510, p=0.001$, respectively). The ROC curves of

148 admission random glucose, fasting glucose, and HbAlc in the nondiabetic patients $(\mathrm{n}=212)$ and

149 patients with diabetes $(n=272)$ are presented in Figures $1 B$ and $1 C$, respectively. In the

150 nondiabetic patients, fasting glucose was not a significant predictor of poor neurological outcomes

$151(\mathrm{AUC}=0.593,95 \% \mathrm{CI}=0.524-0.660, p=0.071)$ with an optimal cutoff value of $109 \mathrm{mg} / \mathrm{dL}$

152 (sensitivity $=39.6 \%$, specificity $=84.8 \%$ ), whereas admission random glucose and HbA1c were 
153 nonsignificant predictors of poor neurological outcomes (AUCs: admission random glucose =

$1540.569,95 \% \mathrm{CI}=0.499-0.636, p=0.129 ; \mathrm{HbA} 1 \mathrm{c}=0.510,95 \% \mathrm{CI}=0.440-0.579, p=0.837)$. In

155 the patients with diabetes, fasting glucose was a significant predictor of poor neurological

156 outcomes $(\mathrm{AUC}=0.596,95 \% \mathrm{CI}=0.535-0.655, p=0.014)$ with an optimal cutoff value of 140

$157 \mathrm{mg} / \mathrm{dL}$ (sensitivity $=62.9 \%$, specificity $=54.5 \%$ ), whereas admission random glucose and HbA1c

158 were nonsignificant predictors of poor neurological outcomes (AUCs: admission random glucose

$159=0.544,95 \% \mathrm{CI}=0.483-0.604, p=0.247 ; \mathrm{HbA1c}=0.503,95 \% \mathrm{CI}=0.442-0.564, p=0.948)$.

160 The AUCs of glycemic measures were not significantly different between the nondiabetic patients

161 and those with diabetes (AUCs: admission random glucose $=0.569$ vs. $0.544, p=0.671$; fasting

162 glucose $=0.593$ vs. $0.596, p=0.963 ; \mathrm{HbA} 1 \mathrm{c}=0.510$ vs. $0.503, p=0.911)$.

163 Table 2 presents the results of univariate and multivariate logistic regression analyses of

164 variables potentially predicting poor neurological outcomes. In univariate logistic regression

165 analysis, age, the NIHSS score at admission, atrial fibrillation, and fasting glucose were significant

166 predictors of poor neurological outcomes in the study patients (crude OR for fasting glucose $=$

$1671.005,95 \% \mathrm{CI}=1.002-1.008, p=0.003)$. However, neither admission random glucose nor HbA1c

168 were significant predictors of poor neurological outcomes. The adjusted OR of admission random

169 glucose was determined using multivariate logistic regression model 1, which included age, sex,

170 the NIHSS score at admission, atrial fibrillation, and admission random glucose. Age and the 
171 NIHSS score at admission were significant predictors of poor neurological outcomes, and

172 admission random glucose was not associated with poor neurological outcomes in model 1

173 (adjusted OR $=1.002,95 \% \mathrm{CI}=0.999-1.005, p=0.228$ ). The adjusted OR for fasting glucose

174 was determined using multivariate logistic regression model 2, which included age, sex, the

175 NIHSS score at admission, atrial fibrillation, and fasting glucose. Age, the NIHSS score at

176 admission, and fasting glucose were significant predictors of poor neurological outcomes in model

1772 (adjusted OR for fasting glucose $=1.005,95 \% \mathrm{CI}=1.003-1.010, p=0.039$ ). The adjusted OR

178 of HbA1c was calculated using multivariate logistic regression model 3, which included age, sex,

179 the NIHSS score at admission, atrial fibrillation, and HbA1c. Age and the NIHSS score at

180 admission were significant predictors of poor neurological outcomes, and HbAlc was not

181 associated with poor neurological outcomes in model 3 (adjusted $\mathrm{OR}=1.160,95 \% \mathrm{CI}=0.985-$

$1821.366, p=0.076)$.

183 Subgroup univariate and multivariate logistic regression analyses were performed in the

184 nondiabetic patients and patients with diabetes (Table 2). In the univariate logistic regression

185 analysis, age, the NIHSS score at admission, atrial fibrillation, and fasting glucose were significant

186 predictors of poor neurological outcomes (crude OR for fasting glucose: nondiabetic patients =

$1871.025,95 \% \mathrm{CI}=1.009-1.041, p=0.002$; patients with diabetes $=1.004,95 \% \mathrm{CI}=1.001-1.008$,

$188 p=0.003)$. However, after adjustment for the influence of age, sex, the NIHSS score at admission, 
189 and atrial fibrillation, all three glycemic measures were not associated with poor neurological

190 outcomes in both groups.

191

192 Discussion

193 In the present study, of the three glycemic measures, fasting glucose was an independent predictor

194 and the most predictive factor of poor neurological outcomes in patients with acute ischemic

195 stroke. ROC analysis revealed that admission random glucose was a predictor of poor neurological

196 outcomes in all study patients; however, it was not associated with poor neurological outcomes in

197 the logistic regression analysis. Furthermore, ROC and logistic regression analyses revealed that

$198 \mathrm{HbA1c}$ was not a predictive factor of poor neurological outcomes. The predictive value of

199 glycemic measures for poor neurological outcomes did not differ significantly between the

200 nondiabetic patients and patients with diabetes.

201 ROC analysis revealed that fasting glucose and admission random glucose have more

202 predictive value than HbA1c does for poor neurological outcomes in acute ischemic stroke. Studies

203 have reported that a high level of fasting or random glucose is associated with poor neurological

204 outcomes or mortality in acute ischemic stroke (Capes et al., 2001; Fang et al., 2013; Hu et al.,

205 2012), and hyperglycemia at the acute stage of the disease may be largely caused by stress

206 (Douketis, 2002). By contrast, HbA1c represents average glycemic levels in the preceding 6-8 
207 weeks and is not affected by transient hyperglycemia; therefore, HbA1c has been perceived as a

208 marker for prestroke glycemic control (Kamouchi et al., 2011; Lattanzi et al., 2016). Furthermore,

$209 \mathrm{HbA1c}$ has been associated with increased mortality (Wu et al., 2014) and poor neurological

210 outcomes (Gao et al., 2016; Hjalmarsson et al., 2014; Lattanzi et al., 2016) in acute ischemic

211 stroke; however, it has also been reported to be nonpredictive of neurological outcomes (Shin et

212 al., 2015). Although many studies have reported the role of hyperglycemia in predicting poor

213 neurological outcomes in acute ischemic stroke, the inclusion criteria for patients, definition of

214 hyperglycemia, and outcome measures varied between these studies; therefore, the results are

215 difficult to compare. Moreover, no study has compared the predictive performance of different

216 definitions of hyperglycemia for neurological outcomes. The results of our study revealed that

217 stress hyperglycemia contributed more than prestroke glycemic control did in predicting negative

218 outcomes in acute ischemic stroke. The ROC analysis revealed that the AUCs of fasting glucose

219 and admission random glucose did not differ significantly; nevertheless, the AUC of fasting

220 glucose was the highest among the three glycemic measures. Furthermore, multivariate logistic

221 regression analysis revealed that fasting glucose was the only independent predictor of poor

222 neurological outcomes among the three glycemic measures (Table 2). Therefore, we speculate that

223 fasting glucose is superior to admission random glucose in predicting neurological outcomes.

224 However, why fasting glucose was more predictive than admission random glucose in this study 
225 remains uncertain, and further investigations are warranted.

226 Some studies have reported that in acute ischemic stroke, hyperglycemia is more detrimental

227 in nondiabetic patients than in patients with diabetes (Capes et al., 2001; Stead et al., 2009; Yao et

228 al., 2016); however, the present findings are not consistent with the aforementioned results,

229 possibly because of the differences in study designs, statistical methods, definition of

230 hyperglycemia, and functional outcome measures. Most studies have used a cutoff value of blood

231 glucose as the criterion for defining hyperglycemia, such as fasting glucose $>140 \mathrm{mg} / \mathrm{dL}$ (Woo et

232 al., 1990) or nonfasting glucose $>130 \mathrm{mg} / \mathrm{dL}$ (Stead et al. 2009). However, we used ROC analysis

233 instead of a cutoff value because of the lack of a widely accepted criterion for defining

234 hyperglycemia in acute ischemic stroke. Moreover, ROC analysis enables the comparison of the

235 predictive performance of different glycemic measures and provides information on sensitivity

236 and specificity, which cannot be obtained by defining hyperglycemia on the basis of a cutoff value.

237 In preclinical animal studies, controlling hyperglycemia with insulin resulted in

238 heterogeneous neuroprotection outcomes (MacDougall \& Muir, 2011). In randomized controlled

239 trials of intensive insulin treatment for glycemic control in patients with acute ischemic stroke,

240 there was no difference in death or dependency between the treatment and control groups, and the

241 results were the same in subgroup analyses of patients with diabetes and nondiabetic patients

242 (Bellolio et al., 2014). Therefore, the pathophysiology of hyperglycemia in acute ischemic stroke 
243 probably involves other factors in addition to high blood glucose. Stress-related cytotoxic

244 neurotransmitters, inflammation, or insulin resistance could be associated with an increased risk

245 of poor neurological outcomes and mortality in patients with stroke and hyperglycemia (Arenillas

246 et al., 2007; Harada et al., 2012; Kruyt et al., 2010). The clinical guidelines published by the

247 American Heart Association/American Stroke Association and the European Stroke Organization

248 recommend that the glycemic levels of patients with acute ischemic stroke be maintained below

$249180 \mathrm{mg} / \mathrm{dL}$. This recommendation is based on the consensus of experts because evidence does not

250 support maintaining the blood glucose at a specific level improves outcomes (European Stroke

251 Organisation Executive \& Committee, 2008; Jauch et al., 2013). Therefore, although

252 hyperglycemia has been confirmed to be a predictor of negative outcomes in stroke, additional

253 investigations are warranted for elucidating the underlying mechanisms and treatment strategies.

254 The present study had some limitations. First, most patients enrolled had mild to moderate

255 initial stroke severity (median NIHSS score at admission $=4, \mathrm{IQR}=2-8$ ), and the influence of

256 hyperglycemia might be more prominent in patients with severe stroke. However, only 84 patients

257 in the present study had an NIHSS score at admission $\geq 10$, and a subgroup analysis of these

258 patients would not have yielded sufficient statistic power. Second, mortality was not included as

259 an outcome because the timing of and reason for mortality could not be assessed accurately in the

260 retrospective medical review. Some patients might have died or received follow-up in facilities 
261 other than the two hospitals included in the present study; therefore, the patients who died or were

262 lost to follow-up within 3 months after stroke were not included in this study. Because of the

263 aforementioned limitations, the results of this study cannot be generalized to all ischemic stroke

264 patients. Third, diabetes was defined as $\mathrm{HbA} 1 \mathrm{c}<6.5 \%$ or as medication usage; therefore, patients

265 with diabetes who were on dietary modification alone with satisfactory HbAlc may have been

266 misclassified as nondiabetic patients.

267

\section{Conclusions}

269 Fasting glucose is an independent predictor of poor neurological outcomes in patients with acute

270 ischemic stroke, with more predictive power than that of admission random glucose and $\mathrm{HbAlc}$.

271 The predictive value of glycemic measures for poor neurological outcomes in acute ischemic

272 stroke did not differ significantly between the nondiabetic patients and those with diabetes.

273 


\section{References}

275

276

277

278

279

280

281

282

283

284

285

286

287

288

289

290

291

292

293

294

295

296

297

298

299

300

301

302

303

304

305

306

307

308

309

Adams HP, Jr., Bendixen BH, Kappelle LJ, Biller J, Love BB, Gordon DL, and Marsh EE, 3rd. 1993. Classification of subtype of acute ischemic stroke. Definitions for use in a multicenter clinical trial. TOAST. Trial of Org 10172 in Acute Stroke Treatment. Stroke 24:35-41.

Ahmed N, Davalos A, Eriksson N, Ford GA, Glahn J, Hennerici M, Mikulik R, Kaste M, Lees KR, Lindsberg PJ, Toni D, and Investigators S. 2010. Association of admission blood glucose and outcome in patients treated with intravenous thrombolysis: results from the Safe Implementation of Treatments in Stroke International Stroke Thrombolysis Register (SITS-ISTR). Arch Neurol 67:1123-1130. 10.1001/archneurol.2010.210

Arenillas JF, Moro MA, and Davalos A. 2007. The metabolic syndrome and stroke: potential treatment approaches. Stroke 38:2196-2203. 10.1161/STROKEAHA.106.480004

Bellolio MF, Gilmore RM, and Ganti L. 2014. Insulin for glycaemic control in acute ischaemic stroke. Cochrane Database Syst Rev:CD005346. 10.1002/14651858.CD005346.pub4

Capes SE, Hunt D, Malmberg K, Pathak P, and Gerstein HC. 2001. Stress hyperglycemia and prognosis of stroke in nondiabetic and diabetic patients: a systematic overview. Stroke $32: 2426-2432$.

DeLong ER, DeLong DM, and Clarke-Pearson DL. 1988. Comparing the areas under two or more correlated receiver operating characteristic curves: a nonparametric approach. Biometrics 44:837-845.

Douketis J. 2002. Review: stress hyperglycemia after ischemic stroke indicates a greater risk for death in patients without diabetes. ACP J Club 136:114.

European Stroke Organisation Executive C, and Committee ESOW. 2008. Guidelines for management of ischaemic stroke and transient ischaemic attack 2008. Cerebrovasc Dis 25:457-507. 10.1159/000131083

Fang Y, Zhang S, Wu B, and Liu M. 2013. Hyperglycaemia in acute lacunar stroke: a Chinese hospital-based study. Diab Vasc Dis Res 10:216-221. 10.1177/1479164112459663

Fuentes B, Castillo J, San Jose B, Leira R, Serena J, Vivancos J, Davalos A, Nunez AG, Egido J, Diez-Tejedor E, and Stroke Project of the Cerebrovascular Diseases Study Group SSoN. 2009. The prognostic value of capillary glucose levels in acute stroke: the GLycemia in Acute Stroke (GLIAS) study. Stroke 40:562-568. 10.1161/STROKEAHA.108.519926

Gao Y, Jiang L, Wang H, Yu C, Wang W, Liu S, Gao C, Tong X, Wang J, Jin Y, and Wu J. 2016. Association between Elevated Hemoglobin A1c Levels and the Outcomes of Patients with Small-Artery Occlusion: A Hospital-Based Study. PLoS One 11:e0160223. 10.1371/journal.pone.0160223 
310 Harada S, Fujita-Hamabe W, and Tokuyama S. 2012. Ischemic Stroke and Glucose Intolerance:

311

312

313

314

315

316

317

318

319

320

321

322

323

324

325

326

327

328

329

330

331

332

333

334

335

336

337

338

339

340

341

342

343

344

345

a Review of the Evidence and Exploration of Novel Therapeutic Targets. Journal of Pharmacological Sciences 118:1-13. 10.1254/jphs.11R04CR

Hjalmarsson C, Manhem K, Bokemark L, and Andersson B. 2014. The role of prestroke glycemic control on severity and outcome of acute ischemic stroke. Stroke Res Treat 2014:694569. 10.1155/2014/694569

Hu GC, Hsieh SF, Chen YM, Hsu HH, Hu YN, and Chien KL. 2012. Relationship of initial glucose level and all-cause death in patients with ischaemic stroke: the roles of diabetes mellitus and glycated hemoglobin level. Eur J Neurol 19:884-891. 10.1111/j.14681331.2011.03647.x

Jauch EC, Saver JL, Adams HP, Jr., Bruno A, Connors JJ, Demaerschalk BM, Khatri P, McMullan PW, Jr., Qureshi AI, Rosenfield K, Scott PA, Summers DR, Wang DZ, Wintermark M, Yonas H, American Heart Association Stroke C, Council on Cardiovascular N, Council on Peripheral Vascular D, and Council on Clinical C. 2013. Guidelines for the early management of patients with acute ischemic stroke: a guideline for healthcare professionals from the American Heart Association/American Stroke Association. Stroke 44:870-947. 10.1161/STR.0b013e318284056a

Kamouchi M, Matsuki T, Hata J, Kuwashiro T, Ago T, Sambongi Y, Fukushima Y, Sugimori H, Kitazono T, and Investigators FSR. 2011. Prestroke glycemic control is associated with the functional outcome in acute ischemic stroke: the Fukuoka Stroke Registry. Stroke 42:2788-2794. 10.1161/STROKEAHA.111.617415

Kruyt ND, Biessels GJ, Devries JH, and Roos YB. 2010. Hyperglycemia in acute ischemic stroke: pathophysiology and clinical management. Nat Rev Neurol 6:145-155. 10.1038/nrneurol.2009.231

Lattanzi S, Bartolini M, Provinciali L, and Silvestrini M. 2016. Glycosylated Hemoglobin and Functional Outcome after Acute Ischemic Stroke. J Stroke Cerebrovasc Dis 25:17861791. 10.1016/j.jstrokecerebrovasdis.2016.03.018

MacDougall NJ, and Muir KW. 2011. Hyperglycaemia and infarct size in animal models of middle cerebral artery occlusion: systematic review and meta-analysis. J Cereb Blood Flow Metab 31:807-818. 10.1038/jcbfm.2010.210

Paciaroni M, Agnelli G, Caso V, Corea F, Ageno W, Alberti A, Lanari A, Micheli S, Bertolani L, Venti M, Palmerini F, Billeci AM, Comi G, Previdi P, and Silvestrelli G. 2009. Acute hyperglycemia and early hemorrhagic transformation in ischemic stroke. Cerebrovasc Dis 28:119-123. 10.1159/000223436

Parsons MW, Barber PA, Desmond PM, Baird TA, Darby DG, Byrnes G, Tress BM, and Davis SM. 2002. Acute hyperglycemia adversely affects stroke outcome: a magnetic resonance 
346

347

348

349

350

351

352

353

354

355

356

357

358

359

360

361

362

363

364

365

366

367

368

369

370

371

imaging and spectroscopy study. Ann Neurol 52:20-28. 10.1002/ana.10241

Ribo M, Molina C, Montaner J, Rubiera M, Delgado-Mederos R, Arenillas JF, Quintana M, and Alvarez-Sabin J. 2005. Acute hyperglycemia state is associated with lower tPA-induced recanalization rates in stroke patients. Stroke 36:1705-1709. 10.1161/01.STR.0000173161.05453.90.9f

Scott JF, Robinson GM, French JM, O'Connell JE, Alberti KG, and Gray CS. 1999. Prevalence of admission hyperglycaemia across clinical subtypes of acute stroke. Lancet 353:376377.

Shin SB, Kim TU, Hyun JK, and Kim JY. 2015. The Prediction of Clinical Outcome Using HbAlc in Acute Ischemic Stroke of the Deep Branch of Middle Cerebral Artery. Ann Rehabil Med 39:1011-1017. 10.5535/arm.2015.39.6.1011

Stead LG, Gilmore RM, Bellolio MF, Mishra S, Bhagra A, Vaidyanathan L, Decker WW, and Brown RD, Jr. 2009. Hyperglycemia as an independent predictor of worse outcome in non-diabetic patients presenting with acute ischemic stroke. Neurocrit Care 10:181-186. 10.1007/s12028-008-9080-0

Woo J, Lam CW, Kay R, Wong AH, Teoh R, and Nicholls MG. 1990. The influence of hyperglycemia and diabetes mellitus on immediate and 3-month morbidity and mortality after acute stroke. Arch Neurol 47:1174-1177.

Wu S, Wang C, Jia Q, Liu G, Hoff K, Wang X, Wang A, Wang C, Zhao X, Wang Y, Liu L, and Wang Y. 2014. HbA1c is associated with increased all-cause mortality in the first year after acute ischemic stroke. Neurol Res 36:444-452. 10.1179/1743132814Y.0000000355

Yao M, Ni J, Zhou L, Peng B, Zhu Y, Cui L, and investigators S. 2016. Elevated Fasting Blood Glucose Is Predictive of Poor Outcome in Non-Diabetic Stroke Patients: A Sub-Group Analysis of SMART. PLoS One 11:e0160674. 10.1371/journal.pone.0160674 


\section{Figure Legend}

373 Receiver operating characteristic (ROC) curves of admission random glucose, fasting glucose, 374 and $\mathrm{HbA} 1 \mathrm{c}$ for predicting poor neurological outcomes (modified Rankin Scale $=3$, 4, or 5 at 3

375 months) in (A) all study patients, (B) nondiabetic patients, and (C) patients with diabetes

$376 * p<0.05$

377 


\section{Figure 1 (on next page)}

\section{Figure 1}

Receiver operating characteristic (ROC) curves of admission random glucose, fasting glucose, and HbAlc for predicting poor neurological outcomes (modified Rankin Scale $=3,4$, or 5 at 3 months) in (A) all study patients, (B) nondiabetic patients, and (C) patients with diabetes. * $p<0.05$ 
(A)

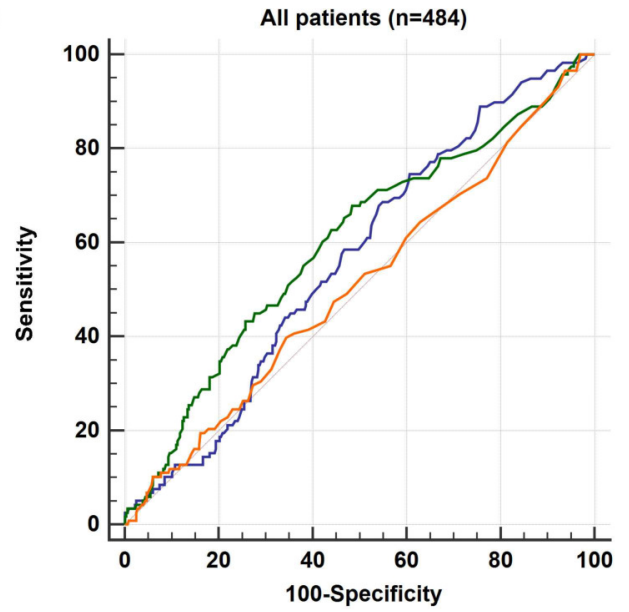

- Random, AUC=0.564 (0.519-0.609, $\left.p=0.026^{*}\right)$ Fasting, AUC $=0.598\left(0.553-0.642, p=0.001^{*}\right)$ HbA1c, AUC $=0.510(0.465-0.556, p=0.742)$
(B)

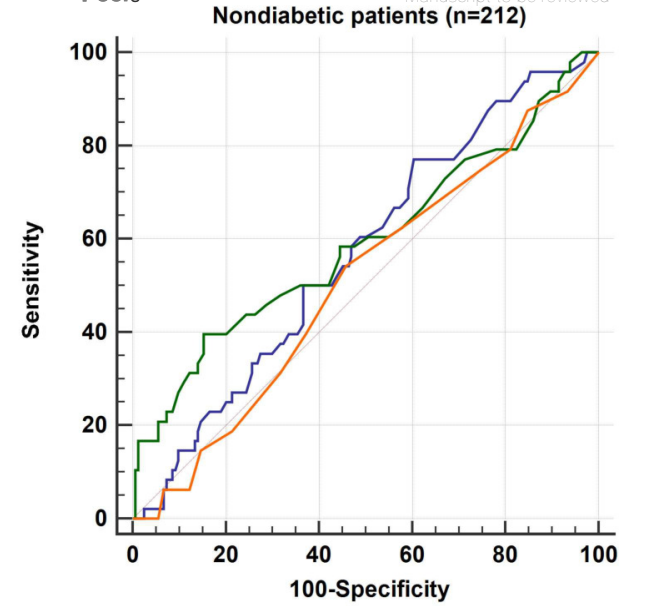

Random, AUC $=0.569(0.499-0.636, p=0.129)$ Fasting, AUC $=0.593(0.524-0.660, p=0.071)$
HbA1c, AUC $=0.510(0.440-0.579, p=0.837)$
(C)

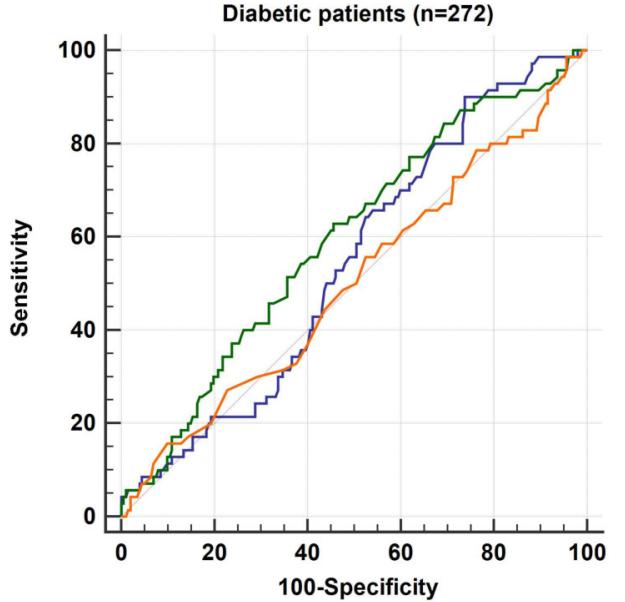

Random, AUC $=0.544(0.483-0.604, p=0.247)$ Fasting, AUC $=0.596\left(0.535-0.655, p=0.014^{\star}\right)$
HbA1c, AUC $=0.503(0.442-0.564, p=0.948)$ 
Table $\mathbf{1}$ (on next page)

Table 1. Clinical characteristics of the patients 
Table 1. Clinical characteristics of the patients

\begin{tabular}{|c|c|c|c|c|}
\hline & $\begin{array}{l}\text { Total patients } \\
\quad(n=484)\end{array}$ & $\begin{array}{l}\text { Nondiabetic patients } \\
\qquad(\mathrm{n}=\mathbf{2 1 2})\end{array}$ & $\begin{array}{l}\text { Diabetic patients } \\
\qquad(\mathrm{n}=\mathbf{2 7 2})\end{array}$ & $p$ value \\
\hline Age, mean \pm SD, years & $67.9 \pm 13.7$ & $65.9 \pm 15.3$ & $69.5 \pm 12.2$ & $0.004 *$ \\
\hline Male sex, n (\%) & $315(65.1 \%)$ & $145(68.4 \%)$ & $170(62.5 \%)$ & 0.177 \\
\hline \multicolumn{5}{|l|}{ Comorbidities, n (\%) } \\
\hline Hypertension & $346(71.5 \%)$ & $132(62.3 \%)$ & $214(78.7 \%)$ & $<0.001^{*}$ \\
\hline Diabetes & $272(56.2 \%)$ & $0(0 \%)$ & $212(100 \%)$ & N.A. \\
\hline Hyperlipidemia & $147(30.4 \%)$ & $62(29.2 \%)$ & $85(31.2 \%)$ & 0.635 \\
\hline Atrial fibrillation & $74(15.3 \%)$ & $38(17.9 \%)$ & $36(13.2 \%)$ & 0.155 \\
\hline NIHSS at admission, median (IQR) & $4(2-8)$ & $4(2-8)$ & $4(2-7)$ & 0.972 \\
\hline mRS at 3 months, median (IQR) & $1(1-2)$ & $1(1-2)$ & $1(1-3)$ & 0.654 \\
\hline poor neurological outcome, n (\%) & $118(24.4 \%)$ & $48(22.6 \%)$ & $70(25.7 \%)$ & 0.432 \\
\hline Stroke etiology subtypes, n(\%) & & & & 0.658 \\
\hline Large artery atherosclerosis & $133(27.5 \%)$ & $56(26.4 \%)$ & $77(28.3 \%)$ & \\
\hline Small artery occlusion & $166(34.3 \%)$ & $69(32.5 \%)$ & $97(35.7 \%)$ & \\
\hline cardioembolism & $26(5.4 \%)$ & $12(5.7 \%)$ & $14(5.1 \%)$ & \\
\hline other determined etiology & $1(0.2 \%)$ & $1(0.5 \%)$ & $0(0 \%)$ & \\
\hline undermined etiology & $158(32.6 \%)$ & $74(34.9 \%)$ & $84(30.9 \%)$ & \\
\hline Admission random glucose, mean $\pm \mathrm{SD}, \mathrm{mg} / \mathrm{dL}$ & $175.5 \pm 86.8$ & $129.5 \pm 34.8$ & $211.4 \pm 97.6$ & $<0.001 *$ \\
\hline fasting glucose, mean $\pm \mathrm{SD}, \mathrm{mg} / \mathrm{dL}$ & $132.9 \pm 58.7$ & $102.1 \pm 19.9$ & $156.9 \pm 67.3$ & $<0.001 *$ \\
\hline
\end{tabular}


HbA1c, mean \pm SD, \%

$7.0 \pm 1.8$

$5.8 \pm 0.4$

$8.0 \pm 1.8$

$<0.001 *$

* p<0.05, IQR: interquartile range, SD, standard deviation 


\section{Table 2 (on next page)}

Table 2. Univariate and multivariate logistic regression analyses of cInicical characteristics and glycemic measures to poor neurological outcome 
Table 2. Univariate and multivariate logistic regression analyses of clinical characteristics and glycemic measures to poor neurological outcome

\begin{tabular}{|c|c|c|c|c|c|c|c|c|}
\hline \multirow[b]{3}{*}{ Characteristics } & \multicolumn{8}{|c|}{ Poor neurological outcome (mRS $\geqq 3$ at 3 months) } \\
\hline & \multicolumn{8}{|c|}{ All patients $(n=484)$} \\
\hline & Crude OR (95\% CI) & $p$ value & $\begin{array}{c}\text { Adjusted OR model } 1 \\
(95 \% \text { CI })\end{array}$ & $p$ value & $\begin{array}{c}\text { Adjusted OR model } 2 \\
(95 \% \text { CI })\end{array}$ & $p$ value & $\begin{array}{l}\text { Adjusted OR model } \\
\qquad 3(95 \% \mathrm{CI})\end{array}$ & $p$ value \\
\hline Age & $1.059(1.040-1.080)$ & $<0.001^{*}$ & $1.056(1.032-1.080)$ & $<0.001^{*}$ & $1.059(1.034-1.084)$ & $<0.001 *$ & $1.059(1.034-1.084)$ & $<0.001^{*}$ \\
\hline Male sex & $0.755(0.492-1.159)$ & 0.199 & $0.816(0.472-1.411)$ & 0.467 & $0.811(0.468-1.404)$ & 0.453 & $0.833(0.481-1.443)$ & 0.515 \\
\hline NIHSS at admission & $1.320(1.246-1.398)$ & $<0.001^{*}$ & $1.313(1.237-1.395)$ & $<0.001^{*}$ & $1.312(1.235-1.392)$ & $<0.001 *$ & $1.319(1.241-1.402)$ & $<0.001^{*}$ \\
\hline Hypertension & $1.159(0.726-1.851)$ & 0.535 & & & & & & \\
\hline Hyperlipidemia & $0.768(0.482-1.223)$ & 0.266 & & & & & & \\
\hline Atrial fibrillation & $2.870(1.710-4.817)$ & $0.001 *$ & $1.387(0.679-2.833)$ & 0.370 & $1.373(0.668-2.822)$ & 0.388 & $1.452(0.706-2.986)$ & 0.311 \\
\hline Admission random glucose & $1.002(0.999-1.004)$ & 0.158 & $1.002(0.999-1.005)$ & 0.228 & & & & \\
\hline Fasting glucose & $1.005(1.002-1.008)$ & $0.003 *$ & & & $1.005(1.003-1.010)$ & $0.039 *$ & & \\
\hline \multirow[t]{2}{*}{ HbA1c } & $1.032(0.919-1.158)$ & 0.600 & & & & & $1.160(0.985-1.366)$ & 0.076 \\
\hline & \multicolumn{8}{|c|}{ Nondiabetic patients $(n=212)$} \\
\hline Characteristics & Crude OR (95\% CI) & $p$ value & $\begin{array}{c}\text { Adjusted OR model } 1 \\
(95 \% \text { CI })\end{array}$ & $p$ value & $\begin{array}{c}\text { Adjusted OR model } 2 \\
(95 \% \text { CI })\end{array}$ & $p$ value & $\begin{array}{l}\text { Adjusted OR model } \\
\qquad 3(95 \% \mathrm{CI})\end{array}$ & $p$ value \\
\hline Age & $\begin{array}{c}1.0061(1.033- \\
1.089)\end{array}$ & $<0.001 *$ & $1.065(1.027-1.103)$ & $0.006^{*}$ & $1.067(1.028-1.106)$ & $0.006^{*}$ & $1.066(1.028-1.106)$ & $0.006^{*}$ \\
\hline Male sex & $0.632(0.323-1.233)$ & 0.178 & $0.722(0.292-1.782)$ & 0.479 & $0.711(0.288-1.758)$ & 0.461 & $0.685(0.275-1.702)$ & 0.415 \\
\hline NIHSS at admission & $1.347(1.231-1.473)$ & $<0.001^{*}$ & $1.355(1.229-1.493)$ & $<0.001^{*}$ & $1.345(1.220-1.482)$ & $<0.001 *$ & $1.359(1.232-1.500)$ & $<0.001 *$ \\
\hline Hypertension & $1.013(0.521-1.968)$ & 0.969 & & & & & & \\
\hline Hyperlipidemia & $0.659(0.311-1.394)$ & 0.275 & & & & & & \\
\hline Atrial fibrillation & $2.787(1.313-5.916)$ & $0.008^{*}$ & $1.424(0.498-4.076)$ & 0.510 & $1.334(0.471-3.792)$ & 0.586 & $1.396(0.494-5.859)$ & 0.529 \\
\hline Admission random glucose & $1.003(0.995-1.012)$ & 0.453 & $0.999(0.987-1.012)$ & 0.886 & & & & \\
\hline
\end{tabular}




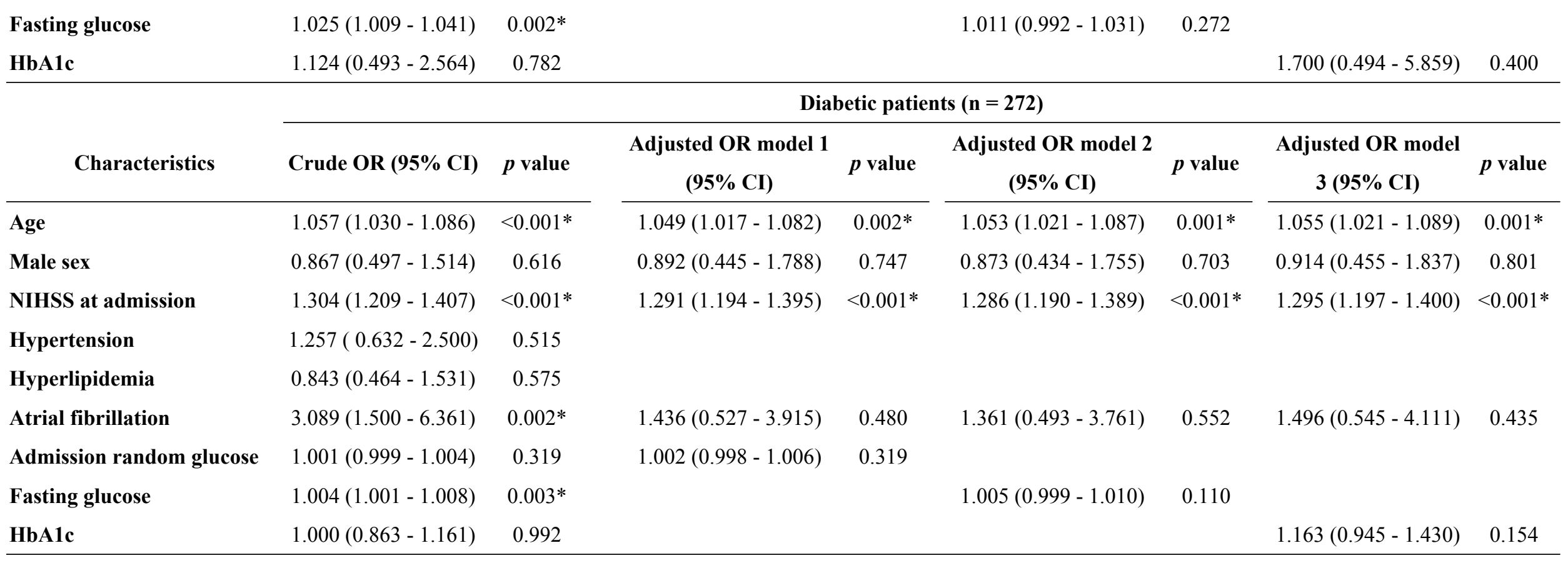

* $\mathbf{p}<0.05$, OR: odds ratio

model 1 included age, sex, NIHSS at admission, atrial fibrillation, and admission random glucose

model 2 included age, sex, NIHSS at admission, atrial fibrillation, and fasting glucose

model 3 included age, sex, NIHSS at admission, atrial fibrillation, and HbAlc 